\title{
Pragmatist Quantum Realism
}

\author{
Richard Healey \\ University of Arizona
}

January, 2018

\begin{abstract}
Realism comes in many varieties, in science and elsewhere. Van Fraassen's influential formulation took scientific realism to include the view that science aims to give us, in its theories, a literally true story of what the world is like. So understood, a quantum realist takes quantum theory to aim at correctly representing the world: many would add that its success justifies believing this representation is more or less correct. But quantum realism has been understood both more narrowly and more broadly.

A pragmatist considers use prior to representation and this has prompted some to dub pragmatist views anti-realist, including the view of quantum theory I have been developing recently. But whether a pragmatist view of quantum theory should be labeled anti-realist depends not only on its ingredients but also on how that label should be applied. Pragmatism offers a healthy diet of quantum realism.
\end{abstract}

\section{Introduction}

Quantum theory has long presented a challenge to a would-be scientific realist who maintains that the historical sequence of successful theories in a mature science like physics offer increasingly accurate representations of a physical world that is largely independent of our observational abilities and practices. Over the past century, quantum theory has amassed an unparalleled record of successful applications over an increasingly wide field of science. But heated controversy continues over whether, and if so how, it represents the world as distinct from our observations of it.

Many philosophers seeking to secure the relation between scientific realism and quantum theory have recently adopted overly narrow conceptions of both relata. The general goal of this paper is to enrich the discussion by noting alternative ways of understanding the project of reconciling quantum theory with scientific realism. My more specific aim is to argue that a pragmatist view of quantum theory facilitates just such a reconciliation.

I begin by building a framework in which to set alternative ways of understanding quantum realism. Besides helping to orient readers who haven't encountered them all, I use this to motivate the pragmatist realism described 
and defended in the bulk of the paper. Section 3 locates a novel form of physical realism recently advocated by Chris Fuchs within this framework, at the same time explaining why his QBist understanding of quantum theory is appropriately regarded as anti-realist.

Then in section 4 I briefly sketch key features of a pragmatist view of quantum theory I have been developing recently. Scientific realism is often taken to be a view or attitude toward how far, and how well, a scientific theory represents the world. But ultimately it is we, as users of theories, who represent the world. In the case of quantum theory, we do so by applying mathematical models to the world. This application involves claims about a target system that are appropriately evaluated for truth and representational accuracy. But, in this pragmatist view, quantum theory itself makes no claim of this form: and scientific realism, broadly construed, does not require a theory to claim that the target system is (more or less) faithfully represented by the model being applied. Section 4 argues that this is not how models of quantum theory are applied.

One symptom of the poverty of recent discussion of scientific realism (at least in the quantum context) is the implicit assumption that any view of a scientific theory is either (narrowly) realist or instrumentalist. In section 5 I review reasons why the pragmatist view just sketched is not instrumentalist. In the following sections of the paper I argue for a conception of scientific realism that deems this pragmatist view realist. Realism about the quantum domain is often taken to require provision of an ontological model. In section 6 I argue that quantum theory does offer such a model in the pragmatist view, despite an unsatisfying incompleteness that may motivate attempts to construct a successor theory.

Section 7 argues that quantum theory offers genuine explanations in the pragmatist view. In section $8 \mathrm{I}$ consider but reject the view that scientific realism involves a thick notion of correspondence truth that is unavailable here.

Section 9 illustrates the limitations of correspondence truth using an interesting recent argument that the universal applicability of quantum theory is incompatible with the assumption that each quantum measurement has a unique, objective outcome. I defend this assumption by appeal to a pragmatist inferential (rather than referential) account of the content of basic representational claims that issue from an application of quantum theory. This account plays a key role in the pragmatist view of section 4 by showing how to eliminate talk of measurement from a precise statement of quantum theory's Born rule without running afoul of "no-go" theorems that refute a naive realist interpretation of quantum theory.

Some follow Putnam in viewing scientific realism as an empirical hypothesis supported by the history of science. Section 10 offers quantum theory as evidence against this hypothesis, suggesting instead that improved symbolic representation of the world be viewed as a long-term scientific aspiration. In conclusion I review the form of quantum realism associated with the pragmatist view of section 4 , pointing out where it diverges from what others have taken quantum realism to involve. 


\section{What is Quantum Realism?}

\section{$2.1 \quad$ Realism}

In approaching the varieties of quantum realism, I'll start with realism in general:

(Realism) The world is the way it is (almost) no matter what anyone may think about it: the only exceptions are processes involved in thinking our thoughts.

A realist about the world of mathematics believes that numbers and their properties exist independently of the existence or thoughts of mathematicians or anyone else. Some mathematical realists take them to exist and have their properties necessarily, so (for example)

$$
5+7=12
$$

would have been true even if there had been no thoughts and even no physical world at all. But if the world contains thoughts, then at least some of its features will depend on whatever is involved in thinking them-hence the qualification.

Quantum realism is concerned with the physical world, not with the world of mathematics. So this gives us

(Physical Realism) The physical world is the way it is (almost) no matter what anyone may think; the only exceptions are physical processes involved in thinking our thoughts.

Our thoughts affect the physical world at least through the actions to which they lead, and this is one way in which physical processes are involved in thinking them (as when my thought of a drink prompts the raising of my glass). For a physicalist this is not the only way, since he believes there is a sense in which our thoughts are physical processes.

\subsection{Scientific Realism}

Physical Realism is a metaphysical thesis. But we ordinarily assume we learn a lot about the world through science. This adds an epistemological, or at least axiological, component to a scientific realism that incorporates Physical Realism. According to Bas Van Fraassen [41], scientific realism is the view that

Science aims to give us, in its theories, a literally true story of what the world is like; and acceptance of a scientific theory involves the belief that it is true. (p. 8)

Such a scientific realist (unlike van Fraassen himself!) takes science to be guided by an epistemic aim that would be achieved only if scientists arrive at literally true theories. Even if the world is ultimately physical, a science 
like psychology or sociology is not primarily concerned with its purely physical aspects: but physics is. Applied to quantum theory, this kind of scientific realist maintains that

Physics aims to give us, in quantum theory, a literally true story of what the physical world is like; and acceptance of quantum theory involves the belief that it is true.

In accordance with Physical Realism, such a literally true story would say what the physical world is like: it would have nothing to say directly about anyone's thoughts or experiences of it. Quantum theory is undoubtedly very successful: there are no empirical reasons to withhold acceptance. This leads to one way of characterizing quantum realism:

(Quantum Scientific Realismo) Quantum theory gives us a literally true story of what the physical world is like.

Scientific realists have a positive epistemic attitude toward the content of our best theories, but the history of physical science cautions against overoptimism. So contemporary scientific realists often restrict their epistemic commitment to the approximate truth of our best theories, despite the obscurity of this notion of approximate truth. Such caution suggests this alternative expression of realism about quantum theory:

(Quantum Scientific Realism) Quantum theory purports to give us a literally true story of what the physical world is like, and we should believe that story is approximately true.

But what is that story? A would-be Quantum Scientific Realist must answer the question "How could the world possibly be the way quantum theory says it is?": she must provide an Interpretation of quantum theory. ${ }^{1}$ Notoriously, this has turned out to be very difficult. Easy answers have been shown to be inadequate, and today the field is littered with a proliferating variety of competing Interpretations of quantum theory in various states of health.

\subsection{Naive Realism}

Many years ago [19] I formulated arguments against what I called Naive Realism, which tells the following story. The quantum domain is supposed to be determinate in that magnitudes always possess definite values on any quantum system. It is held to be objective in that measurement is taken to be merely our way of getting to know what some of these values are. The theory is probabilistic, on this view, because in even the most favorable circumstances there

\footnotetext{
${ }^{1}$ I capitalize the initial ' $I$ ' to distinguish attempts to answer this question within a broader category of attempts to say how quantum theory should be understood. It is a substantial representationalist assumption that the only way to understand quantum theory is to understand how the world could be the way quantum theory says it is.
} 
are magnitudes whose values on a given system at a given time are not derivable from the appropriate quantum mechanical description of the system at that time, while this description does yield probability distributions over sets of distinct values. This simple and inviting form of quantum mechanical realism may be reasonably associated with the names of Popper[32] and Einstein[9]. It may be defined by principles of Precise Values and Faithful Measurement:

(PV) For any quantum system $s$, and any dynamical variable $A$ pertaining to $s$, if $t$ is a time lying within the lifetime of $s$, then $A$ has a unique real value on $s$ at $t$.

(FM) If successful, a measurement of a dynamical variable $A$ pertaining to a quantum system $s$ reveals the pre-existing value of $A$ on $s$.

The arguments were not wholly original: they were based on work by Kochen and Specker [28] and by Bell [5]. The arguments rested on two implicit assumptions.

(Value Independence) The precise value of a dynamical variable is independent of what variable or variables are to be measured.

(Fair Sampling) The probability of success is independent of the value of the measured variable.

The Einstein-Podolsky-Rosen argument [10] may be seen as an argument for Naive Realism, based on their famous Reality Criterion:

If, without in any way disturbing a system, we can predict with certainty (i.e. with probability equal to unity) the value of a physical quantity, then there exists an element of physical reality corresponding to this physical quantity.

In recent discussions of so-called loophole-free tests of local realism ([26], [17], [39]) the term 'realism' is often equated to Naive Realism, with or without some of these other assumptions.

The refutation of Naive Realism is not a death-blow to Quantum Scientific Realism, since Naive Realism is an overly narrow understanding of realism about quantum theory. Would-be scientific realists have implicitly recognized this by investigating the prospects of alternative forms of Quantum Scientific Realism.

\subsection{Wave Function Realism}

One alternative has become known as wave function realism. The name is unfortunate. A wave function $\psi$ is a mathematical, not a physical, entity. (Einstein preferred the more abstract term ' $\psi$-function' while Schrödinger used 'representative', in each case to highlight the object's status as a piece of mathematics.) What a wave function realist is realist about is not this mathematical entity, but 
some physical entity she takes it to represent. Albert ([1], [2]) takes $\psi$ to represent a physical field in a multi-dimensional configuration space; an adherent to the eigenstate-eigenvalue link might identify the physical referents of $\psi$ with the eigenvalue possessed by each variable represented by a self-adjoint operator for which $\psi$ is an eigenvector; a collapse theorist [16] might take $\psi$ to represent mass density, or alternatively flash-propensity [40]; and so on.

The volume entitled "The Wave Function" [3] offers a sampling of recent philosophical work in this tradition that reveals a proliferation of ways of developing, advocating, and criticizing it. As its subtitle reveals, the primary focus of the volume is on metaphysical issues, especially the nature and dimensionality of the space in which the wave function is defined. These are not issues with which physicists are much concerned. As Wallace notes in his contribution to [3], discussions of the metaphysics of wave function realism are often conducted on the basis of assumptions that are hard to square with the way physicists understand currently fundamental forms of quantum theory. Of course there are those who believe that current formulations of these theories are inadequate, so that the term 'quantum theory' as it appears in the thesis of Quantum Scientific Realism must be treated as open to negotiation in the interests of securing the truth of the thesis. ${ }^{2}$ But to arrive at a charitable understanding of the quantum theory we have it seems wise not to adopt a procrustean attitude to that theory in order to fit it to the Interpreter's bed.

\subsection{Spacetime State Realism}

Contemporary formulations of quantum theory do not take the wave function to be the only way to represent a system's quantum state. Even pure states are typically represented by Hilbert space vectors or rays, while mixed states are represented by density operators. In $\mathrm{C}^{*}$-algebraic formulations [38] states are represented not in Hilbert space but by positive, normalized, linear functionals on algebras of observables, and the pure/mixed distinction (differently defined) is supplemented by a further distinction, between normal states and non-normal states that cannot be represented by density operators on a Hilbert space.

After raising objections to wave function realism, Wallace and Timpson [42] instead advocate what they call spacetime state realism as a form of realism about quantum states that better accords with physical practice. For a quantum field theory on a fixed background spacetime, a quantum state is defined on each open region of spacetime, including arbitrary unions of disjoint regions. Ignoring non-normal states, the state of each region may be represented by a density operator on a corresponding Hilbert space. For a quantum spacetime state realist, to specify the state of every open region of spacetime in this way is to give a complete fundamental physical description of world history.

It is not easy to recognize our familiar world in this description, and a proponent of spacetime state realism has his work cut out to convince critics that

\footnotetext{
${ }^{2}$ The work of Bell [5] has been particularly influential in this regard: see the contribution to [3] by Goldstein and Zanghì.
} 
it can be reconstructed from such spare and unfamiliar materials. Wallace and Timpson propose to do this by appeal to decoherence within an Everettian multiverse. But they acknowledge other possible routes, following alternative Interpretational strategies based on hidden variables or dynamical-collapse theories.

\subsection{Should a realist about quantum theory be a Quantum Scientific Realist?}

This section briefly explored a variety of proposed views of quantum theory, each designed to answer the question "How could the world possibly be the way quantum theory says it is?". They by no means exhaust the Interpretations currently on offer, and we can confidently expect new additions. Notoriously, these Interpretations take quantum theory to offer mutually incompatible stories of what the physical world is like. For a constructive empiricist like van Fraassen this presents a problem for physics only to the extent that different Interpretations fail to be empirically equivalent. But the would-be scientific realist cannot be so sanguine. If quantum theory cannot give us a single, literally true story of what the physical world is like then Quantum Scientific Realism cannot be true. To defend realism about quantum theory it seems that one must develop an Interpretation and convince everyone of its superiority over all others, largely if not completely without appeal to experiment. While the arguments have improved over the years, so have the objections against every proposed Interpretation. Perhaps we should think again about what it means to be a realist about quantum theory?

\section{Participatory Realism}

By identifying realism about quantum theory with what I called Quantum Scientific Realism we were led into the morass of rival Interpretations of quantum theory. If we retrace the steps that led us there we arrive once more at what I called

(Physical Realism) The physical world is the way it is (almost) no matter what anyone may think; the only exceptions are physical processes involved in realizing our thoughts.

Frustrated by attempts to label his QBist view of quantum theory instrumentalist or anti-realist, Chris Fuchs has recently classified his view as a form of participatory realism, along with a number of other views of quantum theory.

These views have lately been termed "participatory realism" to emphasize that rather than relinquishing the idea of reality (as they are often accused of), they are saying that reality is more than any third-person perspective can capture. Thus, far from instances of instrumentalism or antirealism, these views of quantum theory should 
be regarded as attempts to make a deep statement about the nature of reality. [15]

By inserting a first-person perspective into the heart of physics, Fuchs portrays QBism as honoring Wheeler's vision of a participatory universe in which "particles" and agents jointly create a universe whose external reality is manifested by the unpredictable experiences that result from each agent's interactions with it.

I see participatory realism as a novel form of Physical Realism. While the physicalist locates my thoughts as ultimately just elements of physical reality, the participatory realist sets them apart in a way that resists inclusion within any merely third-person perspective. So this is not just a novel form of dualism. It is reminiscent of Putnam's ([35], p. xi) (subsequently repudiated) internal realist metaphor

The mind and the world jointly make up the mind and the world.

Taken literally, Fuchs's participatory realism is no metaphor, but the radical metaphysical thesis that reality admits of no third-person view-neither materialist, idealist, nor dualist. As he puts it,

these views of quantum theory should be regarded as attempts to make a deep statement about the nature of reality.

But need a quantum realist accept this, or any similar, metaphysical thesis? Science-based arguments for metaphysical conclusions are notoriously controversial, and in this case so are the arguments for QBism or other participatoryrealist views of quantum theory. ${ }^{3}$ By retreating so far from the morass of Interpretations our would-be quantum realist has backed himself into a dark metaphysical corner!

Moreover, a QBist who endorses this novel form of Physical Realism has totally repudiated the Quantum Scientific Realist demand that quantum theory tell us what the physical world is like. For a QBist, rather than describing the physical world, quantum theory is merely a tool each individual may take up and use to better anticipate that individual's experiences when acting in the physical world. Even after abandoning Quantum Scientific Realism, a scientific realist should expect the practice of quantum physics to yield more substantive information about an independently existing physical world. Surely there's a better way to be a quantum realist?

\section{Representation and a Pragmatist Alternative}

Van Fraassen's formulation of scientific realism arose in the course of his attempt to avoid metaphysics in an account of scientific practice as directed toward epistemological goals. He went on to propose constructive empiricism as a

\footnotetext{
${ }^{3} \mathrm{My}$ [25] provides an introduction to the controversy, including references to relevant literature.
} 
less metaphysically-loaded alternative. Recall the way he formulated scientific realism:

Science aims to give us, in its theories, a literally true story of what the world is like; and acceptance of a scientific theory involves the belief that it is true.

He inserted the word 'literally' to disqualify positivist and other non-literal accounts of theoretical truth. Van Fraassen endorsed the so-called semantic conception of a scientific theory as defined by a class of models, and identified the truth of a theory with the truth of the statement that the world is faithfully represented by some model in this class.

Quantum theory in its various forms is naturally understood to involve a family of mathematical models, some non-relativistic, others relativistic; some used to model systems of particles, others to model systems of fields; etc. Following Van Fraassen's lead, one could then say that, for a scientific realist, to accept quantum theory is to believe that it is literally true - that some model in this family faithfully represents the world. But to say that is to commit once more to the perilous Interpretative quest pursued by the Quantum Scientific Realist.

Here is where pragmatism can offer the would-be quantum realist a better way, by querying the representationalist assumption that a scientific theory can give us a literally true story of what the world is like only by faithfully representing the world. At first sight the realist may see no alternative to this assumption. But an alternative will emerge once we focus on how mathematical models of quantum theory are applied to the world. The key is to see how, in application, a model can guide us toward a literally true story without itself telling it.

I begin with some seemingly pedantic remarks. It is scientists, not science, who create theories even while pursuing scientific aims. And representation is something scientists and other people do with a model, not something the model does by itself. In pursuit of the aims of science, scientists do many things with the theories they create. They apply theoretical models to the world in various ways, including using a model to represent the world or, more typically, a system of interest in the world. Even in a representational application, the model is almost never claimed to offer a faithful representation of the target system, but only to represent it well enough for the purposes at hand. So claims based on application of a theory are almost never given to us by, or in, that theory.

It is distinctive of quantum theory that, when applied to a target system, its models are not applied to represent that system. As I see it, the role of the wave function (or other mathematical representative of a quantum state, a phrase I shall not repeat) is not to describe or represent some physical magnitude, entity or law but to provide good advice to any user of quantum theory about the significance and credibility of magnitude claims about physical systems. This is how a quantum model guides us toward the literal truth of some such claims when the model is applied. Certainly a target system is assigned a 
quantum state, but the primary role of that state is not to represent the system or its properties: it is to permit application of the Born rule to determine what probabilities to assign to various eventualities involving this and similar systems.

I call this the state's primary role because that is the main use of quantum states in physical practice. Physicists usually say that the resulting Born probabilities concern possible measurement outcomes: the demise of naive realism shows they cannot be uniformly understood as probabilities of possessed properties.

But we can and should avoid any talk of measurement by using quantum models of decoherence when determining which properties may be meaningfully ascribed to a system in an application of the Born rule. Ascription of a property to system $s$ is through a magnitude claim of the form $\mathbf{s}$ has $\mathbf{Q} \in \boldsymbol{\Delta}$, where $Q$ is a magnitude such as energy, or a component of position, momentum or spin, and $\Delta$ is a Borel set of real numbers. Models of decoherence may be applied to show that Naive Realism's (PV) is false because there is no physical situation in which, for every such property $\mathbf{Q} \in \boldsymbol{\Delta}$, s may be meaningfully said either to possess, or to lack, that property.

In this way a quantum model can play an important preliminary role by advising a user on how significant is each magnitude claim about a physical system in a particular situation. To assess its significance, the user may apply (unitary) quantum theory to that system in interaction with its environment. In a quantum model of a system and its environment, an initial wave function assigned just to the system would typically evolve extraordinarily rapidly and robustly into a (reduced state) density operator that is extremely close to diagonal in some "pointer basis" determined by the nature of the interaction Hamiltonian. ${ }^{4}$

Only claims concerning each magnitude represented by an operator neardiagonal in the pointer basis are thereby selected as having enough content to be assigned a Born probability, resulting in significant sets of mutually exclusive and jointly exhaustive magnitude claims suitable for application of the Born rule. Anyone who accepts quantum theory should set credence (only) in each significant magnitude claim equal to its Born probability, confident that exactly one claim in the set for each such magnitude is true while the others are false. No magnitude claim that lacks significance in that environmental context is worthy of credence - such meaningless claims cannot even be entertained.

It is on significant magnitude claims that a quantum realist can base a literally true story of what part of the world involving the target system is like, though which story is true will depend on which eventualities actually come to pass. Quantum theory does not tell that story. But we can use quantum theory to reassure ourselves that there is some true story to be told, in terms of true significant magnitude claims and other statements whose truth these claims determine, such as Bell's ([5], p. 52) settings of switches and knobs on experimental equipment, the currents in coils, and the readings of instruments. And we can use quantum theory as a source of good advice on what to expect the true story to be.

${ }^{4}$ For further details, see my [21]. 
The reader will have noticed a certain "fuzziness" in my statement of the pragmatic rules governing the use of the wave function. This is as unproblematic as it is inevitable. Bell [4] began his "Against "measurement"" with the complaint that surely by now we should have an exact formulation of some serious part of quantum mechanics. This may be achieved simply by dropping von Neumann's notorious "projection postulate" (collapse of the wave function on measurement) and removing any reference to measurement, observation, apparatus, classical system, etc. from a statement of the Born rule in the way I have indicated.

Pragmatic rules governing the use of the wave function should not appear in the resulting exact formulation: They concern the application of the theory so formulated. No matter how exactly or precisely a scientific theory is formulated, its application always requires skill and judgment that cannot be made fully explicit. Any pragmatic rule guiding that application remains subject to interpretation by the skilled practitioner. A good physicist is able to judge when it is permissible to apply the Born rule even without deploying a model of environmental decoherence - fortunately, because environments are typically complex open systems for which there are few tractable quantum models of decoherence, and even in these few, completely robust and irreversible diagonalization of system density operator is never more than a very good approximation.

\section{$5 \quad$ Is this Instrumentalism?}

There are realists who will dismiss the pragmatist view briefly introduced in the previous section (and developed further in my [20], [24]) as an unacceptably instrumentalist view of quantum theory. Before going on to argue that this pragmatist view offers a novel form of quantum realism, I'll first respond to this charge of instrumentalism.

According to current usage, instrumentalism in the philosophy of science is the view that a theory is merely a tool for systematizing and predicting our observations: For the instrumentalist, nothing a theory supposedly says about unobservable structures lying behind but responsible for our observations should be considered significant. Moreover, instrumentalists characteristically explain this alleged lack of significance in semantic or epistemic terms: claims about unobservables are meaningless, reducible to statements about observables, eliminable from a theory without loss of content, false, or (at best) epistemically optional even for one who accepts the theory.

But the pragmatist view sketched in the previous section makes no use of any distinction between observable and unobservable structures, so to call it instrumentalist conflicts with current usage. In this pragmatist view, quantum theory does not posit novel, unobservable structures corresponding to quantum states, operators and Born probabilities: these are not beables represented in quantum models. Nevertheless, claims about them in quantum theory are often perfectly significant, and many are true. This pragmatist view does not seek to undercut the semantic or epistemic status of such claims, but to enrich our understanding of their non-representational function within the theory and to 
show how they acquire the content they have.

As we saw in $\S 2.4$, wave function realists take the wave function to represent a novel physical structure-the quantum state-whose existence is evidenced by the theory's success. In this view, a wave function represents a physical structure that either exists independently of the more familiar physical systems to which magnitude claims pertain or else grounds their existence and properties. From this realist perspective, it may seem natural to label as instrumentalist any approach opposed to that account of the quantum state.

But a pragmatist should concede the reality of the quantum state - its existence follows trivially from the truth of quantum claims ascribing quantum states to systems. What he should deny is that a quantum state assignment is true independently of or prior to the true magnitude claims that (in his view) provide the backing for it. Note that any such state assignment is relational, since these are the claims about values of magnitudes accessible from the physical situation of an actual or hypothetical agent that would make this the correct quantum state to assign, relative to that situation. But although they are relational, quantum state assignments are perfectly objective, since this agent-situation is physical and not merely epistemic. (Relational) quantum states could exist even in a world without agents, as long as that world contained physical situations that some agent might have occupied. Under a reasonably expansive conception of agency that will include most or all spatiotemporal worlds.

The truth of a quantum state assignment trivially implies that a wave function represents something we call a quantum state. It does not imply that this quantum state is a beable of quantum theory - a purported element of physical reality that it is the job of the wave function in a quantum model to represent. The previous section described the non-representational roles a wave function plays when a quantum model is applied. In its primary role the wave function offers advice on how strongly to believe magnitude claims. But when a model of quantum theory is applied it is the function of magnitude claims to represent elements of physical reality. These are the claims that underlie statements about the outcomes of quantum measurements, and they play a crucial role in representing what quantum theory is used to predict and explain.

A radical pragmatist might deny that representing reality is ever the primary function of any claim. Such a radical pragmatist could reject the representationalist presupposition of this realist/instrumentalist dilemma-the assumption that mere representation could be a (key) function of an element of theoretical structure that figures centrally in an account of its content. The pragmatist view of quantum theory briefly sketched in $\S 4$ does not require this denial or rejection. But as we'll see in $\S 9$, acceptance of this view of quantum theory does require re-examination of what it is for a magnitude claim to represent physical reality.

Not only does quantum theory inform us about the unobserved: it helps agents improve their beliefs about microscopic phenomena that are often considered unobservable. Many magnitude claims are about properties of systems that are unobservable by unaided human senses. This is to be expected, since 
quantum theory was initially developed as a theory of the microworld, where classical physics was first seen to break down. For the pragmatist, unlike the constructive empiricist or traditional instrumentalist, the observable/unobservable distinction is of no special semantic, epistemic or methodological significance. The use of quantum theory to adjust credences in magnitude claims about microscopic phenomena is not only compatible with the present pragmatist view, but plays an important role in helping us explain regularities they exhibit (see $\S 7)$.

Some may concede that the present pragmatist view of quantum theory is not instrumentalist in the classical sense because it abjures any observable/unobservable distinction. But they may wish to count it as instrumentalist in a more general sense, because it relies on a parallel distinction between quantum and classical, or between quantum states and the magnitudes about whose values these are taken to offer advice.

Certainly, in this pragmatist view, quantum theory itself distinguishes between these magnitudes and quantum states. But the distinction is functional, not epistemic or semantic: and it does not rely on any problematic notion of classicality. The progress of physics has revealed magnitudes unknown to classical physics such as strangeness and (the value of) the Higgs field. Quantum states may offer advice on these magnitudes as well as classical magnitudes such as position and momentum. In an application of a model of quantum field theory, a quantum state can also offer advice on the circumstances in which it is legitimate to make claims about entities, such as Higgs particles or the Higgs field as well as photons and the (classical) electromagnetic field. But quantum states are themselves neither magnitudes nor entities, even when they are real (as follows from the truth of the corresponding quantum state assignments).

Here is how quantum theory relates to the physical world, in the present pragmatist view. In accordance with Physical Realism, the physical world exists and has its properties (almost) independently of the existence, thoughts or activities of human or other agents situated within it. Because of their physical situation, agents like us lack a great deal of information about the world, and especially about how it will develop in our future. By creating physical theories we have been able to improve our imperfect epistemic situation. A theory in classical physics was characteristically applied in claims to the effect that mathematical structures in its models represented physical structures in the world (well enough for the purposes at hand). In this way, the theory was itself taken to represent the world, as containing entities with particular values of physical magnitudes. But an application of a model of quantum theory works differently. The goal is still improved beliefs about the values of physical magnitudes on physical entities, but the theory itself does not represent magnitudes as having values. Instead, it offers advice on which entities can meaningfully be assigned values for particular magnitudes, and what credence should be assigned to possession of different possible values for those magnitudes.

The wave function in a quantum model plays its primary role in issuing this advice by serving as input to the Born rule when the model is legitimately applied. The resulting advice is good just when the correct wave function is 
assigned, in which case the wave function represents the real quantum state of the system to which it is assigned. But correctly representing the quantum state of the system is incidental to the purpose of providing good advice to physically, and so epistemically, limited agents on the values of magnitudes on physical entities. To put it metaphorically, quantum states, though real, would be of no particular interest to an omniscient God with direct epistemic access to the values of all magnitudes on all physical entities throughout world history.

\section{$6 \quad$ No Ontological Model?}

Even if the arguments of the last section convinced you that the pragmatist view briefly sketched in $\S 4$ is not instrumentalist, you may be unwilling to call it realist. In recent foundational discussions, realism has been cast in the framework of ontological models. Here is an example from one recent paper.

(Single-world) Realism: The system has some physical properties, a specification of which is called its ontic state, denoted $\lambda$. Ontic states take values in a (measurable) set called the ontic state space. ([30], p.6)

In non-Everettian versions of wave function realism, a system's ontic state is specified by its wave function $\psi$. In the more general ontological models framework $\lambda$ may or may not include $\psi$, but it may include "hidden" variables (as Bohmian mechanics includes position variables). The motivating thought here is that a realist must be able to tell a story about what is going on in the world in situations where quantum theory makes correct predictions, even though no such story emerges from quantum theory itself (as usually understood).

For one who takes the pragmatist view of $\S 4$, there is a meaningful story to be told about the values of various magnitudes in circumstances when the content of claims about them is well enough defined. But these magnitudes are not always well defined, and so there is no ontic state space in which even one of them always has a precise value. So this view permits only a "gappy" story of what is going on in the world that does not conform to (Single world) Realism. For example, when Bell inequalities are violated in photon experiments there is nothing significant one can say about the polarization properties of each entangled photon prior to detection.

But how reasonable is it to impose (Single-world) Realism as a condition on anything that could count as quantum realism? A "gappy" story is still a story about what the physical world is like, independent of the existence or activities of agents or observers. Accepting this view of quantum theory does not commit one to the belief that the world is mind-dependent in any way that would conflict with Physical Realism.

The story provided by Newton's theory was not rejected as anti-realist despite the fact that it included no mechanism filling the gaps between the sun and the earth on which it exerted a gravitational attraction. Admittedly, its successor (Einstein's theory of general relativity) gave a more complete story with its 
dynamic spacetime permitting continuous propagation of (now confirmed) gravitational waves. A realist may hold out the hope of a similar completion of the "gappy" quantum story, perhaps involving a kind of retrocausation that would undermine the independence condition required to rule out Naive Realism.[33]

\section{Is this Pragmatist view Explanatory?}

For a realist, the demand for an ontological model is closely associated with the need to provide genuine understanding. Bell, for example, maintained that only if reformulated precisely in terms of a clear ontology of "beables" could quantum theory supply the kind of explanations we need to understand the big world outside the laboratory. How genuine is the understanding provided by a "gappy" story that is all quantum theory permits on the pragmatist view?

As I have argued elsewhere ([20], [22], [24]), in this pragmatist view quantum theory helps us to explain a host of otherwise puzzling phenomena by showing that they were to be expected and what they depend on. The primary target of explanation is not individual events but what I call probabilistic phenomena. A probabilistic phenomenon is a probabilistic data model of a statistical regularity. To explain a probabilistic phenomenon using quantum theory one locates it within a general class of similar phenomena and shows how the probability distributions in each case are a consequence of a similar legitimate application of the Born rule to a quantum state that is correctly assigned to the systems concerned. This unifies all phenomena in this class by providing something close to what Railton [37] called a deductive-nomological probabilistic (DNP) explanation of each phenomenon.

Here are my reasons for the qualification. Unlike Railton, I consider the primary explanandum in each case to be not an individual chance event but the probabilistic phenomenon such events manifest. For Railton, a DNP explanation of an individual event must allude to a (probabilistic) causal mechanism that gives rise to that event. But while use of quantum theory to explain a probabilistic phenomenon must display an appropriate dependence of events manifesting it, the form of that dependence need not be causal; and even when it is, the term 'mechanism' does not seem apt. Here are two examples to illustrate each point.

That individual hydrogen atoms are stable against spontaneous collapse is a (probability 1) example of a probabilistic phenomenon. We can use quantum theory to explain this phenomenon by showing that the expectation value of a hydrogen atom's energy has a finite lower bound, so the probability is zero for it to have the arbitrarily large negative energy associated with collapse. No causal mechanism is appealed to in the explanation. That hydrogen atoms manifest this phenomenon (by not collapsing) does not depend on a causal mechanism that produces it but on their constitution: each is constituted by an electron and a proton interacting through an electromagnetic potential.

A violation of a Bell inequality is a probabilistic phenomenon. We can use quantum theory to explain this phenomenon by deriving the relevant proba- 
bility distributions from the Born rule, legitimately applied to the appropriate polarization-entangled state of photon pairs whose detection manifests the phenomenon. The events of polarization detection in an individual pair depend causally on whatever events (described by magnitude claims about preparation devices) back the assignment of that entangled state, since interventions on those devices would alter their probabilities. But the distant events of polarizationdetection in each instance are causally independent. Moreover, quantum theory has nothing to say about any continuous causal process mediating the causal dependence of an individual detection event on its backing conditions, and Bell's theorem shows that these events can be produced by no causal mechanism of a kind we have previously encountered.

Some realists may not be satisfied by such explanations on the grounds that they yield only partial understanding. But this is not sufficient reason to reject the view of quantum theory that lay behind them as not realist. In this pragmatist view, quantum theory helps us explain these phenomena by appeal to what is going on in the physical world with no reference to observers, agents or minds. A realist who hankers after more may be compared to Newton, who remained dissatisfied with explanations using his own theory of universal gravitation and continued to search fruitlessly for "the cause of gravity". Over 200 years passed before Einstein's general relativity permitted the reconceptualization that since gravity is not a force it needs no "cause". Dissatisfaction with the good explanations quantum theory helps us to give is not a reason to reject the pragmatist view of quantum theory, though it may motivate the search for a theory that can help us give more satisfying explanations.

\section{Truth}

In this pragmatist view, quantum theory helps us describe the physical world by means of magnitude claims, on whose significance and credibility it offers us advice. These are what we use to make statements about physical reality when applying a quantum model, and their truth or falsity is what we ultimately care about. Realism is often associated with a correspondence theory of truth, so it is important to address the objection that the way the pragmatist view treats magnitude claims is incompatible with correspondence truth and therefore also quantum realism.

Recall that, in this view, the significance of a magnitude claim about a system depends on the system's environment. Consider the claim $C: \mathbf{s}$ has $\mathbf{Q} \in \boldsymbol{\Delta}$. This attributes property $\mathbf{Q} \in \boldsymbol{\Delta}$ to $s$. As Tarski insisted, $C$ is true if and only if $s$ has $\mathbf{Q} \in \boldsymbol{\Delta}$, and that will be so just in case ' $s$ ' refers to $s$ and $s$ satisfies ' $\mathbf{Q} \in \boldsymbol{\Delta}$ '. The pragmatist readily assents to this Tarskian demand, and if that is all that correspondence truth comes to then correspondence truth is part of this pragmatist view. But some scientific realists demand more of correspondence truth. Field [11] further demanded a physicalist reduction of reference relations, and even self-avowed pragmatist Philip Kitcher once argued that 
Reference relations are causal relations between mind-independent entities and linguistic tokens. ([27], p.347)

However, there seem to be cases in which linguistic tokens succeed in referring to mind-independent entities incapable of bearing causal relations, as when I use the word 'one' or the numeral ' 1 ' to refer to the number 1 . Of course nominalists deny the existence of numbers and other non-physical entities. But one who adopts this pragmatist view of quantum theory is already committed to the reality of quantum states and Born probabilities to which we succeed in referring even though these don't enter into causal relations.

I agree with Stephen Leeds [29] that a quantum realist can and should reject a "thick" notion of correspondence truth that requires reference to be understood as a causal relation in favor of a more minimal account of truth and reference. This is not the place to survey the several deflationary options currently on offer. I merely insist that whatever version of minimalism is adopted should be accompanied by an account of the wider function of attributions of truth and reference capable of explaining the importance of these concepts in our discourse. This seems especially important in an era of "alternative facts"!

In quantum theory, claims about quantum states and Born probabilities guide belief about the magnitude claims that are basic to the theory's use in predicting, explaining and controlling phenomena. From this perspective it is natural to think that terms appearing in magnitude claims refer to their subject matter in a way that is somehow more immediate or concrete than terms like 'probability' or 'wave function'. But I think it would be a mistake for a realist to be misled by this thought into adopting a causal account of how reference works in the case of magnitude claims. Indeed, in the next section I will show how a recent argument undermines any such "thick" notion of correspondence even for claims about the outcomes of quantum measurements whose truth some true magnitude claims determine.

\section{The Limits of Quantum Objectivity}

Realists may disagree about whether wave functions represent something physically real, whether electrons have precise positions and momenta, and whether the world is non-local. But on one point all (except Everettians) agree: quantum measurements each have a unique, physically real outcome and their statistics are correctly predicted by quantum theory. A recent argument by Frauchiger and Renner [13] seeks to show that this assumption is inconsistent with the universal applicability of quantum theory itself. The original argument is too long to repeat here, so instead I shall sketch a simpler version I first heard in a talk by Matthew Pusey.

Consider the following (completely unrealizable!) thought-experiment. Suppose that Alice and Bob decide to conduct measurements of various polarization components on a large number of pairs of photons, where each pair is correctly assigned the same polarization-entangled state. Being lazy, they do not at first 
perform any measurements themselves, but delegate that task to their friends, Carol and Dan respectively, each of whom performs the required measurements in his or her otherwise completely physically isolated laboratory. For each pair of photons, Carol measures polarization of one photon with respect to axis $c$ while Dan measures polarization of the other photon with respect to axis $d$. By assumption, each of their measurements has a unique, physically real outcome (as registered in their notebooks or stored in their computers): and quantum theory correctly predicts the correlations between these outcomes from the joint probability distribution $P(c, d)$ calculated by application of the Born rule to the entangled state assigned to the pairs (where $c, d$ are random variables whose values indicate the outcome of the respective polarization measurement).

After each photon pair is measured by Carol and Bob, instead of asking them what outcomes they observed, Alice and Bob apply very carefully tailored interactions to the entire contents $C, D$ of their respective laboratories (including Carol and Dan themselves). They do this repeatedly, for each photon pair independently. Quantum theory then predicts the effect of these interactions is to restore each photon pair to its original entangled state and to restore each lab+occupant $C, D$ to its state prior to the polarization measurement, thus permitting Carol and Bob to continue their measurements. Finally, Alice measures polarization of one photon in each pair with respect to axis $a$ while Bob measures polarization of the other photon with respect to axis $b$.

By assumption, each of Alice's and Bob's measurements also has a unique, physically real outcome (as registered in their notebooks or stored in their computers): and quantum theory correctly predicts the correlations between these outcomes from the joint probability distribution $P(a, b)$ calculated by application of the Born rule to the same entangled state assigned to the pairs. Given our working assumption, quantum theory also correctly predicts the correlations between Carol's outcomes and Bob's from the probability distribution $P(b, c)$, and between Alice's outcomes and Dan's from the probability distribution $P(a, d)$, each of which may again be calculated by applying the Born rule to the same entangled state assigned to the pairs.

If the entangled photon state and the axes $a, b, c, d$ are chosen appropriately, the probabilistic correlations predicted in this way by quantum theory will violate a Bell inequality (the so-called CHSH inequality). But since they constitute a joint distribution over all four measured variables the statistics of these assumed real outcomes will always conform to the inequality. We have a contradiction. So the assumption is false: quantum measurements do not always have unique, physically real outcomes whose statistics are correctly predicted by quantum theory. But predictions of quantum theory have always been confirmed by the statistics of measurement outcomes. So we cannot assume that these measurement outcomes are uniquely physically real!

For one who adopts the pragmatist view of $\S 4$, a quantum measurement has a unique, physical outcome. A statement about that outcome has a determinate, mind-independent truth-value: its truth hinges on that of magnitude claims about physical systems involved in the measuring apparatus. This pragmatist view not only escapes refutation in the imagined scenario, but also receives sup- 
port from its deeper analysis. The key point is that, in this view, acceptance of quantum theory modifies the content of a statement about the outcome of a quantum measurement by restricting what inferences may legitimately made from its truth. The restriction effectively relativizes that content to the environmental context of the system to which quantum theory may be applied. That content then becomes a function of the physical environment within which the system is located. The way content depends on physical environment may be modeled by a quantum model of decoherence.

In all practicably realizable circumstances the environmental context is appropriately modeled by massive decoherence of the relevant quantum state, so that all physically situated agents (not only human agents like Alice, Bob and friends) are able to neglect the fact that content may depend on environmental context and successfully attribute a context-independent content to a statement about any measurement outcome. But in the (completely practicably unrealizable) circumstances described in the thought-experiment this is not so, since Alice, Bob and friends do not share a single environmental context. In these circumstances it is investigators' labs that provide the relevant environmental context. Here context-relativity can be indicated by an appropriate subscript. For example, the content of a magnitude claim $M$ reporting the outcome of one of Carol's measurements on a system in her lab $C$ may be represented as $[M]_{C}$. In their situation they (and we) may continue to agree that there are true statements about their unique physical measurement outcomes with objective, mind-independent content. But that content is not context-independent since it does not license reliable inferences between different environmental contexts.

Decoherence confined to each of their individual laboratories models the environmental context underlying the content of each claim about the outcome of a measurement in that lab. For Carol and Dan physically to have exchanged information they would have had to physically combine their environmental contexts to form a unified context $C \cup D$ into which their statements about their outcomes could have been reliably exported. Alternatively, either Carol or Dan might have physically exchanged information with Alice or Bob without first exchanging information with each other, permitting each reliably to export statements about his or her outcomes into that incompatible larger context. But in fact no such physical interactions occurred in the imagined scenario, in which the environments $C, D$ remained sealed off from each other and also from the environments $A, B$ of Alice and Bob. The upshot is that while the content of each statement about the unique outcome of every measurement on each of an entangled pair of photons may be treated here as perfectly well defined within an environmental context, there is no such context in which a statement about all these outcomes has well defined content.

When Alice and Bob combine their results in their physically unified joint environment $A \cup B$, statements of the outcomes $a, b$ modeled by the joint distribution $P(a, b)$ are significant in $A \cup B$, and the Born rule may legitimately be applied to (correctly) predict this joint distribution. But the absence of the required unified contexts renders illegitimate any application of the Born rule to predict the joint probabilities $P(c, d), P(b, c), P(a, d)$ that also appear in a 
statement of the CHSH inequality. Indeed, by applying quantum theory in this scenario we can see that the CHSH inequality cannot be derived here since there is no environmental context in which its constituent probabilities are all well defined. So statements of the unique, contextually well-defined, physical outcomes of all quantum measurements in the imagined scenario violate no legitimately derivable Bell inequality.

There is a common philosophical view of how content depends on context that may appear to be in tension with the idea that content can depend on context in this way. In this view, a statement has significant content if and only if it expresses a determinate proposition. While what content a statement expresses may depend on the context to which it relates (loosely, to the context in which it is made), context merely determines what proposition a claim expresses. Any variation of content with context can be represented by a function from context into proposition expressed.

In this view, an adequate analysis of a statement's content must then supply an account of the content of each proposition in the range of that function in a referential semantics that provides its truth conditions: if the function is only partial, the claim has no content in a context in which it expresses no determinate proposition. So an adequate analysis of the content of a statement $S$ reporting the outcome of a measurement will either assign it some specific content (varying from context to context) or no content at all (in other contexts). It follows that no analysis is adequate according to which what varies with context is not simply the specific content of the statement but also how much content it has.

This philosophical view provides an idealized model of content that is helpful in elucidating the meaning of indexicals like 'I' and 'now', for which the context in which a statement is made seems readily specifiable (by saying who made it and when). It becomes problematic in circumstances where contextual elements are harder to pin down. As an example, consider the statement $K$, when uttered on a road trip across Kansas

$K$ : Kansas is flatter than a pancake.

What determinate proposition does this express? $K$ may be taken literally or as a metaphor for the literal claim

$V$ : Kansas is very flat.

Taking it literally, intrepid researchers [12] compared a geographic profile of the state based on a digital elevation model provided by the United States Geographic Survey to the profile of a particular pancake from the International House of Pancakes using a confocal laser microscope. They chose as a measure of flatness the deviation from sphericity of an ellipsoid, and estimated this in each case from a best fit to two chosen orthogonal transects of the surface. On this measure, Kansas proved to be much flatter than the pancake: the authors concluded that: 
The calculated flatness of the pancake transect from the digital image is approximately 0.957 , which is pretty flat, but far from perfectly flat. Kansas's flatness is approximately 0.9997. That degree of flatness might be described, mathematically, as "damn flat."

The latter claim presumably establishes the truth of $V$. But on an alternative, qualitative local measure of flatness the authors commented that

When viewed at a scale of $50 \mathrm{~mm}$, a pancake appears more rugged than the Grand Canyon.

One might quibble that Kansas cannot be flat since its elevation varies from 4039 feet to 679 feet above sea level, or that (as noted in one YouTube video taken from a speeding car) you can see the curvature of the earth. You see the problem: When uttered on a road trip across Kansas, the context fails to pick out any well defined proposition expressed by statement $K$, even though that statement clearly conveys something that is both significant and true.

It may be tempting to dismiss such difficulties in specifying the context of utterance for claims like $K$ and $V$ as arising from their vague or metaphorical language. But on closer examination the same kind of problems afflict even the paradigm case of the indexical 'now'. For what exactly is it to specify the time at which a statement is uttered? Any actual utterance is not an event but a process extending over an interval of time. But even precisely to specify an instant within this process either (falsely) presupposes a universal absolute time or (assuming relativity theory) requires a further specification of a state of motion associated with a local inertial frame and/or a spacelike hypersurface including some point-event in the utterer's vicinity.

One committed to a propositional model of content might acknowledge the resulting indeterminacy in exactly what proposition is expressed by utterance of a sentence including the indexical 'now', while maintaining that essentially the same content is conveyed by the utterance for every admissible way of resolving this indeterminacy. But that would commit her both to a non-propositional notion of content and to the task of explaining why the contingent circumstances of human communication render this propositional indeterminacy harmless. Such contingencies have been explored by both philosophers and physicists.

Butterfield [8] notes the importance of several physical features of the environment in which we generally communicate, including the proximity of the parties and the slow rate at which the timely topics of their communication change, compared to the speed of their communication. By considering relativistic physics, Hartle [18] and Penrose [31] (pp. 392-3) note the further importance of the slow relative speed of communicators. Any attempt precisely to specify what proposition is expressed by utterance of a sentence including a temporal indexical like 'now' would require careful application of physical theories to the environment of utterance. But an account of its content does not require this. Even a qualitative analysis generally suffices to explain how such a tensed utterance is able to convey content that is sufficiently well defined for practical human purposes. 
Returning to the imagined scenario of the thought-experiment, what proposition is picked out by Alice's statement that the outcome of the 100th of her measurements was a detection of a photon horizontally polarized with respect to axis $a$ ? In the pragmatist view of $\S 4$, the truth value of this statement in context is determined by that of some magnitude claim of the form $M$ : s has $\mathbf{Q} \in \boldsymbol{\Delta}$. One can give an account of the truth conditions of a claim of the form $M: \mathbf{s}$ has $\mathbf{Q} \in \boldsymbol{\Delta}$ but this is trivial. For example: $M$ is true if and only if the system to which ' $s$ ' refers has a value for the magnitude to which ' $Q$ ' refers that lies in the set of real numbers to which ' $\Delta$ ' refers. Once the (tensed) claim is somehow(!) indexed to a time, these truth conditions are independent of context, since the claim contains no explicit indexical elements.

The problem with this referential approach is not that it is wrong but that, once one has despaired of a physicalist or causal account of reference, it is too shallow to be helpful. The approach fails to illuminate the different ways a claim of the form $M$ functions when $s$ is in different environments. The claim functions within a web of inferences, and the extent of its content depends on the context provided by the presence of other claims in the web-here, an assumption about $s$ 's quantum state is required in assessing the content of a claim of the form $M$ about $s$, since assignment of a (reduced) quantum state reflects the extent and nature of environmental decoherence in a quantum model of Alice's environment A.

The referential approach to content sometimes provides a useful analytic model of content, but it lacks the resources to account for how content accrues to a statement. To understand quantum theory one needs to adopt a better account of what gives a statement content. By modifying inferential relations involving magnitude claims quantum theory affects their content, rendering this contextual. Philosophers customarily regard a claim as meaningful if and only if it expresses a definite proposition when made in an adequately specified context: otherwise it is taken to be meaningless. An improved pragmatist inferentialist approach to the content of an empirical claim (Brandom [6], [7]) accepts a role for context but replaces this "digital" view of content with an "analog" view. Content accrues by degrees as links are added to the inferential web within which statements are located. By making the content of a magnitude claim about a system a function of the environment, acceptance of quantum theory cautions one against taking that claim to attribute an intrinsic property to an object independently of environmental context, even while insisting on the objective content of the claim.

It is only because the situation of agents like us in the physical world is such that we will always inevitably share a single "decoherence environment" that we are able to ignore the implicit dependence on our physical situation of the contents, not only of statements about the outcomes of quantum measurements, but of practically all claims about macroscopic, and most claims about microscopic, systems. 


\section{Scientific Realism as an Empirical Thesis}

Hilary Putnam ([34], [36]) viewed scientific realism as an empirical hypothesis that we should accept as the best, if not the only, explanation of the success of science. He endorsed a formulation of Richard Boyd as influencing his conception of scientific realism - that terms in a mature science typically refer, and theories accepted in a mature science are typically approximately true. His famous "no miracle" argument took the success of science and the preservation of terms like 'electron' through scientific theory change as evidence for scientific realism, so conceived. The realist explanation of these features of science (success and reference preservation) is that scientists mirror the world - in the sense of constructing symbolic representations of their environment - and that science succeeds in the way it does because these symbolic representations become increasingly accurate as science progresses.

Faced with potential counterexamples from the history of science, this empirical hypothesis has been clarified or modified in two ways. Some have sought to distinguish terms for a theory's working posits, whose successful reference is supported by its success, from other non-referring terms. Supporters of structural scientific realism have taken refuge from apparent counterexamples in an appeal to theoretical preservation of representations of structure, rather than (the nature of) objects, with the progress of science. A recent paper by Steven French [14] adopts this perspective toward quantum theory.

Which features of a scientific theory should a scientific realist take to represent the world? Answer: those features that are responsible for the theory's explanatory successes. When the theory is quantum mechanics, the wave function is surely one of those features. ([3], p. 76)

But, in the present pragmatist view, what is distinctive about the success of quantum theory is precisely that it is not due to introduction of new symbols (for beables) to represent novel structures in the physical world. Quantum theory introduces terms like 'wave function' and 'observable' with a different function. They are not intended to mirror the physical world but to guide scientists and other situated agents in better deploying representational resources they already have or are engaged in developing. Even if an important long-term scientific aim is improved symbolic representation of the physical world, science may at times progress faster by introducing key terms without that representational function. Language and other symbolic systems provide scientists and the rest of us with wonderful tools for achieving our goals. But these tools don't always function in the same way. Perhaps the central pragmatist moral of quantum theory is that scientists may find new ways of furthering long-term scientific aspirations by creating theories whose key terms do not function as representations of physical reality. 


\section{How to be a Quantum Realist}

I have advocated a certain conception of scientific realism and argued that by taking a pragmatist view one can see that quantum theory fits this conception. A scientific realist should at least accept the existence of a physical world that is largely independent of how we think of or observe it. So scientific realism incorporates what I called Physical Realism. Quantum theory is compatible with Physical Realism, in the pragmatist view of $\S 4$. While agreeing that there is a physical world whose existence is independent of how we think of it, a Fuchsian participatory realist takes its development to be sensitive to our thoughts through our intentional actions that precipitate an unpredictable observed response. While I see this as a novel form of Physical Realism, others may count it a rejection of this minimal scientific realist requirement.

As an epistemological optimist, a scientific realist should further maintain that through our best science we have learned a lot about what the physical world is like, including many of its features that we cannot observe through our unaided senses. Applications of quantum theory have certainly led to successful predictions and explanations of physical phenomena, many of which are in this sense unobservable. The pragmatist view of $\S 4$ helps us understand how we apply quantum theory in deepening our knowledge of these phenomena without taking wave functions or other elements of its mathematical models to represent beables. But for a QBist quantum theory has taught us little or nothing about what the physical world is like: it has merely provided each agent with a tool that is valuable in anticipating that agent's future experiences when acting in the physical world.

The most direct way a scientific theory could teach us about the physical world is by itself describing it, or offering mathematical models by which to represent it. For a Quantum Scientific Realist, that is the only way we could learn from quantum theory about the physical world. Adopting this restricted conception of quantum realism has led to a proliferation of rival Interpretations of quantum theory, each eager to extract a literally true story of what the physical world is like from quantum theory itself. But pragmatism encourages a wider perspective on how we might gain knowledge of the physical world. A pragmatist who takes the view of $\S 4$ rejects Quantum Scientific Realism. She sees quantum theory as a radically different kind of theory that teaches us about the world not by offering models by which to represent it, but by advising us on how it may meaningfully be represented, and how likely is each meaningful representation to be true. This is how quantum theory has taught us a lot, not only about what the world is like but also about the scope and limitations of our representations of it.

A scientific realist should reject a "thick" correspondence view of truth in favor of a deflationary account, and regard referential semantics as offering an analytic model of content rather than a substantive account of its origins. These pragmatist attitudes to truth and reference underlie $\S 4$ 's pragmatist view of quantum theory. By adopting an inferentialist account of how content accrues to a statement, this pragmatist view shows how to eliminate all Bell's [5] (p.215) 
proscribed words from a formulation of quantum theory with no measurement problem while yielding a statement of the Born rule compatible with the "no-go" theorems of [5], [28]. It also shows how to reconcile the existence of a unique, objective, physical outcome of each successful quantum measurement with the argument of [13].

William James presented his pragmatism as a conciliatory view in philosophy. I think of $\S 4$ 's pragmatist view of quantum theory as offering to reconcile the views of Bohr and Einstein. If Bohr was right that acceptance of quantum theory requires acknowledgment of the limits this puts on our abilities to speak meaningfully about the physical world, perhaps Einstein was right to hold out the hope that these limits may be transcended as quantum theory is succeeded by an even more successful theory that gives us an approximately true, literal story of what the physical world is like.

\section{Acknowledgements}

Thanks to David Glick, Doreen Fraser, an anonymous referee and the editors. I hope your comments and queries have helped me express the views of this paper more clearly even where they differ from your own!

\section{References}

[1] Albert, D. [1996] "Elementary Quantum Metaphysics", in Cushing, J., Fine, A. and Goldstein, S. eds., Bohmian Mechanics and Quantum Theory: An Appraisal. (Dordrecht: Kluwer), pp. 277-84.

[2] Albert, D. [2013] "Wave Function Realism", in Albert, D. and Ney, A. eds. [2013], pp. 52-57.

[3] Albert, D. and Ney, A. eds. [2013] The Wave Function: Essays on the Metaphysics of Quantum Mechanics. (New York: Oxford University Press).

[4] Bell, J.S. "Against 'Measurement'" [1989], reprinted in Bell, J.S. [2004], pp. 213-31.

[5] Bell, J.S. [2004] Speakable and Unspeakable in Quantum Mechanics, 2nd edition. (Cambridge: Cambridge University Press).

[6] Brandom, R. [1994] Making It Explicit. (Cambridge, MA: Harvard University Press).

[7] Brandom, R. [2000] Articulating Reasons: An Introduction to Inferentialism. (Cambridge, MA: Harvard University Press).

[8] Butterfield, J. [1984] "Seeing the Present", Mind, 93, pp. 161-176.

[9] Einstein, A. [1949] "Reply to Criticisms", in P.A. Schilpp, ed. Albert Einstein, Philosopher-Scientist. (Illinois: Open Court), pp. 665-688. 
[10] Einstein, A., Podolsky, B. and Rosen, N. [1935] "Can Quantum-Mechanical Description of Physical Reality be Considered Complete?" Physical Review 47, pp. $777-80$.

[11] Field, H. [1972] "Tarski's Theory of Truth", The Journal of Philosophy, 69, pp. 347-375.

[12] Fonstad, M., Pugatch, W. and Vogt, B. [2003] "Kansas is Flatter than a Pancake", Annals of Improbable Research, 9, pp. 16-18.

[13] Frauchiger, D. and Renner, R. [2016] "Single-world Interpretations of Quantum Theory Cannot be Self-consistent", arXiv 1604.07422 [quant-ph].

[14] French, S. [2013] "Whither Wave Function Realism?", in Albert and Ney [2013], pp. 76-90.

[15] Fuchs, C. [2016] "On Participatory Realism", arXiv:1601.04360v3 [quant$\mathrm{ph}$.

[16] Ghirardi, G.-C., [2017] "Collapse Theories", The Stanford Encyclopedia of Philosophy (Spring 2016 Edition), Edward N. Zalta (ed.), URL = $<$ https://plato.stanford.edu/archives/spr2016/entries/qm-collapse/>.

[17] Giustina, M., et al. [2015]. "Significant-Loophole-Free Test of Bell's Theorem with Entangled Photons". Physical Review Letters, 115, 250401.

[18] Hartle, J. [2005] "The Physics of Now", American Journal of Physics 73, pp. 101-9.

[19] Healey, R. [1979] "Quantum Realism: Naïveté is no Excuse", Synthese, 42, pp. 121-44.

[20] Healey, R. [2012] "Quantum Theory: A Pragmatist Approach", The British Journal for the Philosophy of Science, 63, pp. 729-71.

[21] Healey, R. [2012a] "Quantum Decoherence in a Pragmatist View: Dispelling Feynman's Mystery", Foundations of Physics 42, pp. 1534-1555.

[22] Healey, R. [2015] "How Quantum Theory Helps us Explain", The British Journal for the Philosophy of Science, 66, pp. 1-43.

[23] Healey, R. [2016] "Local Causality, Probability and Explanation", in Bell, M. and Gao, S. eds. Quantum Nonlocality and Reality 50 Years of Bell's Theorem. (Cambridge: Cambridge University Press), pp. 172-194.

[24] Healey, R. [2017] The Quantum Revolution in Philosophy. (Oxford: Oxford University Press). 
[25] Healey, R. [2017a] "Quantum-Bayesian and Pragmatist Views of Quantum Theory", The Stanford Encyclopedia of Philosophy (Spring 2017 Edition), Edward N. Zalta (ed.), URL = $<$ https://plato.stanford.edu/archives/spr2017/entries/quantumbayesian $/>$.

[26] Hensen, B. et al. [2015] "Loophole-free Bell inequality violation using electron spins separated by 1.3 kilometres", Nature 526, pp. 682-686.

[27] Kitcher, P. [2002] "On the Explanatory Role of Correspondence Truth", Philosophy and Phenomenological Research, 64, pp. 346-64.

[28] Kochen, S. and Specker, E. [1967] "The problem of hidden variables in quantum mechanics", Journal of Mathematics and Mechanics 17, pp. 5987.

[29] Leeds, S. [2007] "Correspondence Truth and Scientific Realism", Synthese, 159, pp. 1-21.

[30] Leifer, M. and Pusey, M. [2017] "Is a Time-symmetric Interpretation of Quantum Theory Possible without Retrocausality?", arXiv 1607.07871v2 [quant-ph].

[31] Penrose, R. [1989] The Emperor's New Mind. (Oxford: Oxford University Press).

[32] Popper, K. R. [1967] "Quantum Mechanics Without 'The Observer', in M. Bunge, ed. Quantum Theory and Reality. (Berlin: Springer).

[33] Price, H. and Wharton, K. [2016] "Taming the quantum spooks", Aeon. https://aeon.co/essays/can-retrocausality-solve-the-puzzle-of-action-at-adistance

[34] Putnam, H. [1978] Meaning and the Moral Sciences. (London: Routledge and Kegan Paul).

[35] Putnam, H. [1981] Realism, Truth and History. (Cambridge: Cambridge University Press).

[36] Putnam, H. [2012] Philosophy in an Age of Science. (Cambridge, Mass.: Harvard University Press).

[37] Railton, P. [1978] "A Deductive-Nomological Model of Probabilistic Explanation", Philosophy of Science, 45, pp. 206-226.

[38] Ruetsche, L. [2011] Interpreting Quantum Theories (Oxford: Oxford University Press).

[39] Shalm, L. K. et al. [2015] "Strong Loophole-Free Test of Local Realism", Physical Review Letters 115, 250402. 
[40] Tumulka, R. [2006] "Collapse and relativity", in A. Bassi et al. eds. Quantum mechanics: Are there quantum jumps? and On the present status of quantum mechanics. AIP Conference Proceedings, 844, 340-51.

[41] Van Fraassen, B. [1980] The Scientific Image. (Oxford: Clarendon Press).

[42] Wallace, D. and Timpson, T. [2010] "Quantum Mechanics on Spacetime I: Spacetime State Realism", The British Journal for the Philosophy of Science, 61, pp. 697-727. 